\title{
The Strain energy density to estimate lifetime of notched components subjected to variable amplitude fatigue loading
}

\author{
Zheng $\mathrm{Hu}$ \\ Science and Technology on Vebicle Transmission Laboratory, China North Vebicle Research Institute, Beijing, China, 100072 \\ buгbengvip@126.com
}

Filippo Berto

Norwegian University of Science and Technology, NO-7491 Trondheim, Norway

filippo.berto@ntnu.no,http://orcid.org/0000-0001-9676-9970

Luca Susmel

Department of Civil and Structural Engineering, the University of Sheffield, Sheffield S1 3JD, UK

l.susmel@sheffield.ac.uk, bttps://orcid.org/0000-0001-7753-9176

ABSTRACT. In the present paper, the approach based on the strain energy
density (SED) averaged over a structural volume is reformulated to estimate
the lifetime of notched components subjected to variable amplitude (VA)
uniaxial fatigue loading. The accuracy and reliability of the proposed
reformulation of the SED approach was checked against a large number of
data taken from the literature and generated, under two different load spectra,
by testing specimens of carbon steel C40 containing notches of different
sharpness. Such a validation exercise allowed us to demonstrate that the
extension of the SED approach as proposed in the present paper is capable of
accurately estimating fatigue damage in notched components subjected to in-
service VA fatigue loading.

KEYWORDS. Strain energy density; Notch; Variable amplitude loading.

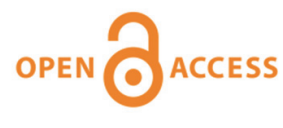

Citation: $\mathrm{Hu}$ Z, Berto F, Susmel L, The Strain energy density to estimate lifetime of notched components subjected to variable amplitude fatigue loading, Frattura ed Integrità Strutturale, 47 (2019) 383-393.

Received: 15.11 .2018

Accepted: 04.12.2018

Published: 01.01.2019

Copyright: (C) 2019 This is an open access article under the terms of the CC-BY 4.0, which permits unrestricted use, distribution, and reproduction in any medium, provided the original author and source are credited.

\section{INTRODUCTION}

$\mathrm{M}$ ost components contain material defects or geometric discontinuities. Examination of the state of the art demonstrate that the effect of stress concentrators on the fatigue strength of engineering materials has been investigated by considering mainly constant amplitude (CA) fatigue situations. However, most components experience variable amplitude (VA) fatigue load histories which make the characteristics of the critical local stress/strain states more complex, with this affecting markedly fatigue strength. 
In order to predict in-service lifetime of notched components under VA loading, it is important to make use of specific methods suitable for taking into account the way fatigue damage accumulates itself. Palmgren [1] and Miner [2] first formulated this concept in a mathematical form as:

$$
D=\Sigma\left(n_{i} / N_{\mathrm{f}, i}\right)
$$

where $D$ denotes the cumulative damage sum, and $n_{i}$ and $N_{\mathrm{f}, i}$ are the applied cycles and the total number of cycles to failure under the $i$ th constant amplitude stress level, respectively. When engineering metallic materials are damaged by VA load histories, failure is supposed to occur as soon as the critical value of cumulative damage sum $D_{\text {cr }}$ is equal to unity. However, experimental evidence under VA loading condition indicates that in situations of practical interest $D_{\text {cr varies in }}$ the range 0.02-5 [3, 4]. Clearly, this fact can produce a remarkable discrepancy between estimated and actual lifetime, with this calculation resulting in non-conservative predictions.

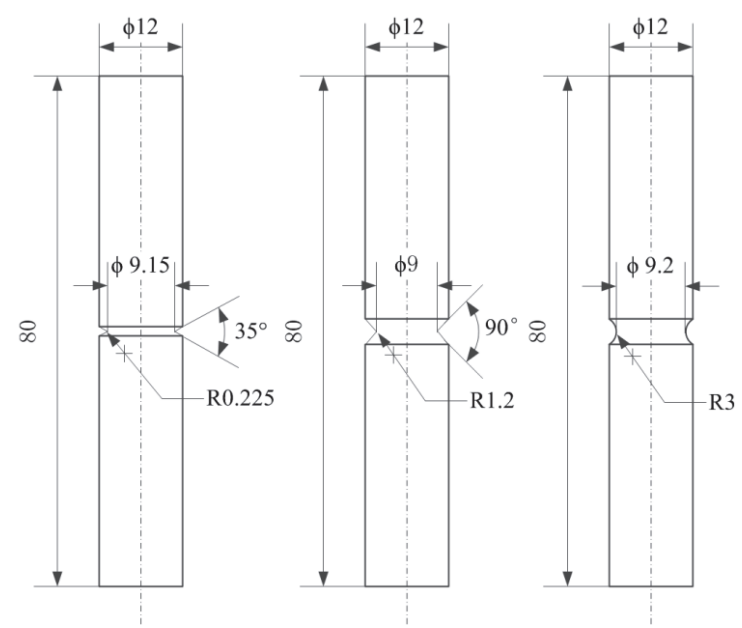

(a)

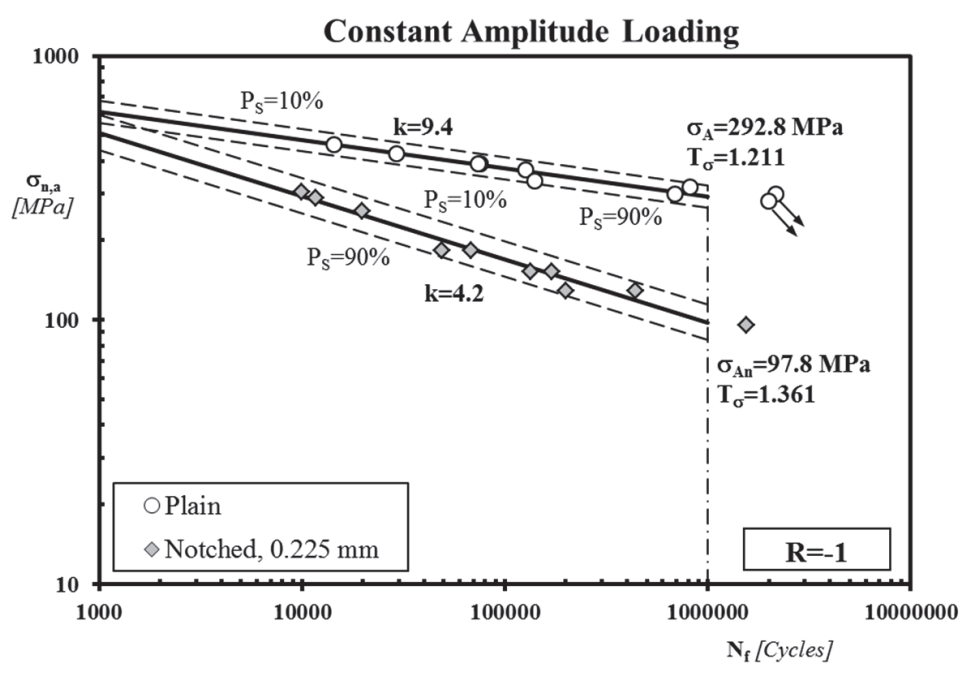

(b)

Figure 1: (a) Geometries of the tested notched samples (dimensions in millimetres). (b) Fatigue results generated under CA uniaxial fatigue loading [3].

Fatigue life estimation techniques based on local approaches require some detailed information about the stress-strain state around the notch tip [3,5-9]. Different SED-based approaches to deal with notch fatigue problems have been formulated since the beginning of the last century. Recently, Lazzarin et al. [8, 10, 11] developed a volume-based approach, in which the SED calculations are carried out in a material-related control volume. The averaged SED approach 
is based both on a precise definition of the control volume and on the fact that the critical energy does not depend on the notch sharpness. The control radius $R_{0}$ of the volume is a material property, which involves the plain specimen's fatigue limit and the threshold stress intensity factor range [10]. The approach was successfully used under both static and CA fatigue loading conditions to assess the strength of notched components subjected to uniaxial [12-14] as well as to multiaxial loading [15-19].

Even if a lot of theoretical and experimental work has been carried out to check the accuracy and reliability of the SED approach, surprisingly, so far no systematic attempt has been made to extend the use of this powerful method to those situations involving VA fatigue loading. Accordingly, the ultimate goal of the research work summarized in the present paper was to reformulate the SED approach to make it suitable for assessing fatigue lifetime of notched components subjected to VA uniaxial fatigue load histories, with this being done by using a suitable cycle counting method (i.e., the Rain-Flow method [20]) as well as a suitable cumulative damage model (i.e., Palmgren and Miner's rule [1, 2]).

\section{EXPERIMENT DETAILS}

$\mathrm{I}$ $\mathrm{n}$ a recent work, Susmel and Taylor [3] tested under uniaxial VA fatigue loading circumferentially notched specimens made of a medium-carbon steel C40 and containing three different geometrical features. The geometry of the tested specimens is reported in Fig. 1a along with details of the notches that were characterized by three different values of the notch tip radius and opening angle. Static tensile tests were performed in order to evaluate the mechanical properties according to the ASTM standard procedure. The yield stress, tensile stress and Young's modulus of the investigated materials were $672 \mathrm{MPa}, 852 \mathrm{MPa}$ and $209000 \mathrm{MPa}$, respectively. The net stress concentration factor, $K_{\mathrm{t}}$, was calculated by solving standard linear-elastic Finite Element (FE) models where the mesh density in the notch region was gradually increased until convergence occurred. This standard numerical procedure returned a $K_{\mathrm{t}}$ value under tension equal to 4.42 , 2.2 and 1.66 for the specimens with a root radius of $0.225,1.2$ and $3 \mathrm{~mm}$, respectively.

Both CA and VA fatigue tests were carried out in axial load control under a nominal load ratio, $R=\sigma_{\min } / \sigma_{\max }$, equal to -1 at a frequency of $4 \mathrm{~Hz}$. All the CA fatigue results are summarised in the Wöhler diagrams of Fig. 1b in terms of nominal stress amplitude evaluated onto the net cross-sectional area of the specimens. Fig. 1b shows the fatigue data generated by testing both the un-notched specimens and the $\mathrm{V}$-notched samples with root radius equal to $0.225 \mathrm{~mm}\left(K_{\mathrm{t}}=4.42\right)$. The statistical analyses have been performed assuming a log-normal distribution of the number of cycles to failure for each stress amplitude. All data obtained from specimens characterized by a fatigue life between $10^{4}$ and $2 \times 10^{6}$ have been taken into account in the statistical analyses. It is worth observing here that the scatter bands plotted in the chart of Fig. $1 \mathrm{~b}$ were calculated for each stress level by assuming a confidence level equal to $95 \%$. The results from the statistical re-analysis are summarised in Tab. 1 in terms of: nominal stress amplitudes ( $\sigma_{\mathrm{A}}$ for the plain specimens and $\sigma_{\mathrm{An}}$ for the notched samples) for a probability of survival $\mathrm{P}_{\mathrm{S}}=50 \%$ at a reference number of cycles to failure, $N_{\mathrm{A}}$, equal to $10^{6}$; inverse slope, $k$, of the Wöhler curves and the scatter index $T_{\sigma}$ that provides the width of the scatter band between the curves with a probability of survival of $10 \%$ and $90 \%$ respectively (with a confidence level equal to $95 \%$ ).

\begin{tabular}{ccccccccc}
\hline Specimen Type & $\mathrm{r}_{\mathrm{n}}(\mathrm{mm})$ & $\mathrm{d}_{\mathrm{g}}(\mathrm{mm})$ & $\mathrm{d}_{\mathrm{n}}(\mathrm{mm})$ & $2 \alpha\left(^{\mathrm{9}}\right)$ & $k$ & $\sigma_{\mathrm{A}}, \sigma_{\mathrm{An}}{ }^{*}(M P a)$ & $T_{\sigma}$ & $K_{\mathrm{t}}$ \\
Plain & - & 12 & 6 & - & 9.4 & 292.8 & 1.211 & 1.0 \\
Sharp & 0.225 & 12 & 9.15 & 35 & 4.2 & 97.8 & 1.361 & 4.42 \\
\hline
\end{tabular}

Table 1: Summary of the experimental results generated under fully-reversed CA loading [3].

As to the performed VA tests, the specimens were tested under the two different random spectra (Fig. 2) having sequence length, $n_{\text {tot }}=\sum n_{\mathrm{i}}$, equal to 1000 cycles. All the random spectra were repeated until failure of the specimens occurred. In particular, the Concave Upwards Spectrum (CUS) was derived from a conventional Rayleigh distribution, whereas the Concave Downwards Spectrum (CDS) was defined so that we could have few cycles with high stress ranges combined with a relatively large number of cycles with low stress ranges [21]. The aim of two different spectra of tests was to determine the effect of spectrum shape on the fatigue lifetime of the notched material being investigated and to determinate the fatigue lifetime under random block loading.

All the results generated by Susmel and Taylor [3] are summarized in Tabs. 2-4, where the performed tests are described in terms of adopted spectrum, maximum force, $\mathrm{F}_{\mathrm{a}, \max }$, maximum amplitude in the spectrum of the axial, $\sigma_{\mathrm{a}, \max }$, load ratio, $\mathrm{R}$, and experimental value of the number of cycles to failure, $N_{\mathrm{f}}$. For comparison, Tab. 5 shows the fatigue results generated by testing plain samples under the two load spectra. Tab. 5 also reports the corresponding experimental values of the critical damage sum, $D_{\text {crexp }}$, calculated by using experimental CA plain Wöhler curve. 


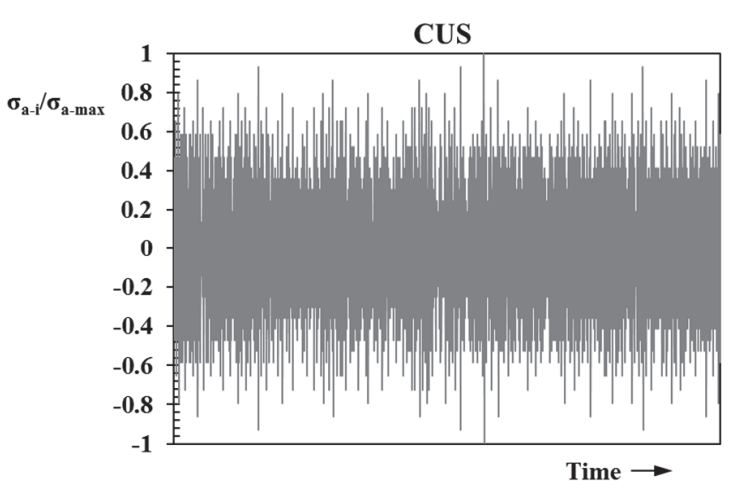

(a)

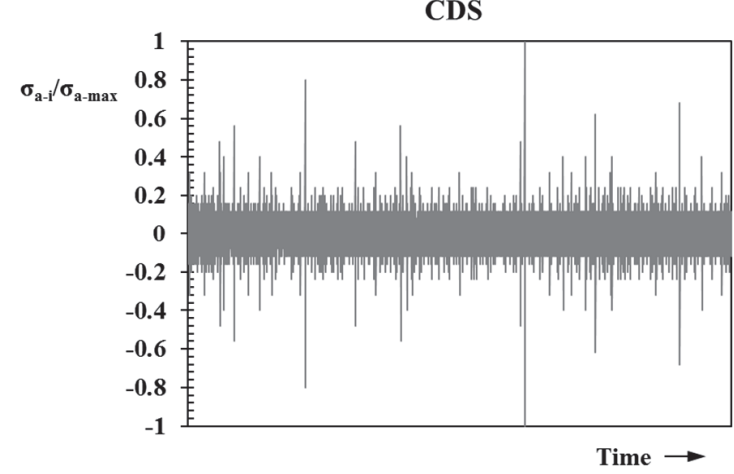

(b)

Figure 2: Adopted load spectra (a) CUS and (b) CDS [3].

\begin{tabular}{rccccc}
\hline Code & Spectrum & $\mathrm{F}_{\mathrm{a}, \max }(\mathrm{kN})$ & $\sigma_{\mathrm{a}, \max }(\mathrm{MPa})$ & $\mathrm{R}$ & $N_{\mathrm{f}}($ Cycles $)$ \\
S2_VA & CUS & 34.2 & 520.1 & -1 & 20975 \\
S3_VA & CUS & 34.2 & 520.1 & -1 & 21568 \\
S4_VA & CUS & 18.4 & 279.8 & -1 & 170155 \\
S6_VA & CUS & 18.4 & 279.8 & -1 & 182155 \\
S7_VA & CUS & 25.6 & 389.3 & -1 & 66703 \\
S9_VA & CUS & 25.6 & 389.3 & -1 & 61594 \\
S10_VA & CDS & 65.8 & 1000.7 & -1 & 8620 \\
S12_VA & CDS & 49.3 & 749.7 & -1 & 45620 \\
S13_VA & CUS & 14.4 & 219.0 & -1 & 391420 \\
S14_VA & CDS & 65.8 & 1000.7 & -1 & 7620 \\
S15_VA & CDS & 49.3 & 749.7 & -1 & 42620 \\
S16_VA & CDS & 35.4 & 538.4 & -1 & 179620 \\
S17_VA & CUS & 14.4 & 219.0 & -1 & 339763 \\
S19_VA & CDS & 28.4 & 431.9 & -1 & 367620 \\
S20_VA & CDS & 35.4 & 538.4 & -1 & 172620 \\
S21_VA & CDS & 28.4 & 431.9 & -1 & 376620 \\
\hline
\end{tabular}

Table 2: Summary of the experimental results generated by testing the notched specimens with root radius equal to $0.225 \mathrm{~mm}$ under VA fatigue loading [3].

\begin{tabular}{cccccc}
\hline Code & Spectrum & $\mathrm{F}_{\mathrm{a}, \max }(\mathrm{kN})$ & $\sigma_{\mathrm{a}, \max }(\mathrm{MPa})$ & $\mathrm{R}$ & $N_{\mathrm{f}}($ Cycles $)$ \\
I1_VA & CUS & 35.5 & 520.4 & -1 & 37568 \\
I2_VA & CUS & 29.5 & 432.4 & -1 & 84463 \\
I3_VA & CUS & 23.5 & 344.5 & -1 & 170581 \\
I4_VA & CUS & 35.5 & 520.4 & -1 & 34044 \\
I5_VA & CUS & 29.5 & 432.4 & -1 & 83663 \\
I6_VA & CUS & 23.5 & 344.5 & -1 & 253927 \\
I7_VA & CDS & 65.4 & 958.6 & -1 & 10620 \\
I8_VA & CDS & 48.5 & 710.9 & -1 & 78620 \\
I9_VA & CDS & 65.4 & 958.6 & -1 & 11905 \\
I10_VA & CUS & 20.5 & 300.5 & -1 & 715859 \\
I11_VA & CDS & 31.0 & 454.4 & -1 & 732620 \\
I12_VA & CDS & 31.0 & 454.4 & -1 & 531620 \\
I13_VA & CDS & 37.0 & 542.4 & -1 & 285277 \\
I14_VA & CUS & 20.5 & 300.5 & -1 & 588009 \\
I15_VA & CDS & 48.5 & 710.9 & -1 & 76620 \\
I16_VA & CDS & 37.0 & 542.4 & -1 & 330620 \\
\hline
\end{tabular}

Table 3: Summary of the experimental results generated by testing the notched specimens with root radius equal to $1.2 \mathrm{~mm}$ under VA fatigue loading [3]. 


\begin{tabular}{cccccc}
\hline Code & Spectrum & $\mathrm{F}_{\mathrm{a}, \max }(\mathrm{kN})$ & $\sigma_{a, \max }(\mathrm{MPa})$ & $\mathrm{R}$ & $N_{\mathrm{f}}($ Cycles $)$ \\
B1_VA & CUS & 42.0 & 631.8 & -1 & 18736 \\
B2_VA & CUS & 42.0 & 631.8 & -1 & 15715 \\
B3_VA & CUS & 33.0 & 496.4 & -1 & 65733 \\
B4_VA & CUS & 24.0 & 361.0 & -1 & 274715 \\
B5_VA & CUS & 27.5 & 413.7 & -1 & 118044 \\
B6_VA & CUS & 24.0 & 361.0 & -1 & 300763 \\
B7_VA & CUS & 33.0 & 496.4 & -1 & 63450 \\
B8_VA & CUS & 27.5 & 413.7 & -1 & 138975 \\
B9_VA & CDS & 65.0 & 977.8 & -1 & 13620 \\
B10_VA & CDS & 49.0 & 737.1 & -1 & 85397 \\
B11_VA & CDS & 39.0 & 586.7 & -1 & 352620 \\
B12_VA & CDS & 39.0 & 586.7 & -1 & 300216 \\
B13_VA & CDS & 65.0 & 977.8 & -1 & 12620 \\
B14_VA & CDS & 33.5 & 503.9 & -1 & 665620 \\
B15_VA & CDS & 33.5 & 503.9 & -1 & 749620 \\
B16_VA & CDS & 49.0 & 737.1 & -1 & 78392 \\
\hline
\end{tabular}

Table 4: Summary of the experimental results generated by testing the notched specimens with root radius equal to $3 \mathrm{~mm}$ under VA fatigue loading [3].

\begin{tabular}{ccccc}
\hline Spectrum & $\sigma_{\mathrm{a}-\max }(\mathrm{MPa})$ & $\mathrm{R}$ & $N_{\mathrm{f}}$ (Cycles) & $D_{\exp }$ \\
CUS & 728.6 & -1 & 14763 & 0.59 \\
CUS & 728.6 & -1 & 13525 & 0.54 \\
CUS & 594.2 & -1 & 49450 & 0.29 \\
CUS & 512.8 & -1 & 264594 & 0.38 \\
CUS & 512.8 & -1 & 219086 & 0.32 \\
CDS & 891.3 & -1 & 32620 & 1.31 \\
CDS & 735.6 & -1 & 136620 & 0.91 \\
CDS & 618.9 & -1 & 356620 & 0.47 \\
CDS & 891.3 & -1 & 23620 & 0.95 \\
CDS & 618.9 & -1 & 380085 & 0.50 \\
& & Average Value $=$ & 0.63 \\
\hline
\end{tabular}

Table 5: Summary of the experimental results generated by testing the plain samples under fully-reversed VA loading and experimental values of the critical damage sum, $D_{\text {cr }}[3]$.

\section{THE SED TO ESTIMATE LIFETIME OF NOTCHED COMPONENTS UNDER CA FATIGUE LOADING}

I $\mathrm{n}$ recent years, the strain energy density (SED) has been widely used to estimate the fatigue lifetime of notched components subjected to CA fatigue loading [10, 12, 13, 22, 23].

The averaged SED approach states that failure occurs when the mean value of the strain energy density averaged over a control volume surrounding the notch tip is equal to a critical energy value. For rounded V-notches, the control volume is as depicted in Fig. 3 , where its radius $R_{0}$ does not depend on the notch geometry. In more detail, $R_{0}$ is the depth of the crescent shape as measured along the notch bisector line. The outer radius of the control volume equals $R_{0}+r_{0}$, where $r_{0}$ is defined as a function of both the notch opening angle $2 \alpha$ and the notch root radius $\rho$, according to the following relationship:

$$
r_{0}=\rho \cdot \frac{\pi-2 \alpha}{2 \pi-2 \alpha}
$$

Dealing with the fatigue assessment of notched components, the control radius, $R_{0}$, can be defined by imposing the equality, with reference to the fatigue limit condition [24], between the averaged SED relevant to the plain specimens and that obtained from $\mathrm{V}$-notched specimens with known geometry, i.e.: 


$$
\Delta W_{0}^{\text {plain }}=\frac{\sigma_{\mathrm{A}, \mathrm{p}}^{2}}{2 E}=\Delta W^{\text {notch }}\left(R_{0}\right)
$$

where $\sigma_{\mathrm{A}, \mathrm{p}}$ is the fatigue strength of the plain sample at $N_{\mathrm{A}}$ cycles and $E$ is the elastic modulus. By varying the control radius $R_{0}$ around the notch tip in an FE model under the nominal stress $\sigma_{\mathrm{A}, \mathrm{n}}$, the corresponding values of strain energy density $\Delta W^{\text {notch }}$ averaged over the control volume can be determined. Then the numerical value $\Delta W^{\text {notch }}\left(R_{0}\right)$ as a function of the control radius $R_{0}$ is generated by a fitting equation. Finally, the control radius $R_{0}$ of the critical volume can be calculated since it is equal to the strain density energy of the plain sample, $\Delta W_{0}^{\text {plain }}$. Due to the absence of experiment data related to stress intensity factor, the control radius can be easily evaluated by the procedure above.

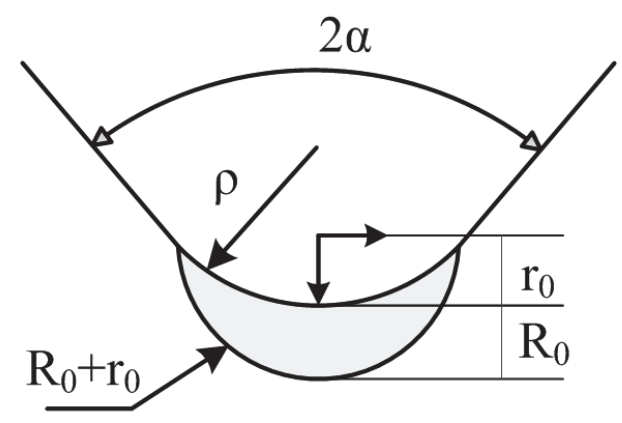

Figure 3: Definition of the control volume for specimens weakened by rounded V-notch.

The averaged SED can also easily and quickly be calculated from FE analyses by summing the energy contributions $W_{\text {element, }}$ for all the finite elements within the control volume $V$, i.e.:

$$
\Delta W=c_{w} \frac{\sum_{V} W_{\text {element }, i}}{V}
$$

Eq. (4) defines the so-called direct approach to evaluate the SED parameter. In order to consider the influence of the nominal load ratio $R$, the weighting parameter $c_{\mathrm{w}}$ has to be adopted by using the following expressions [25, 26]:

$$
c_{w}(R)=\left\{\begin{array}{ccc}
\frac{1+R^{2}}{(1-R)^{2}} & \text { for } & -\infty \leq R<0 \\
1 & \text { for } & R=0 \\
\frac{1-R^{2}}{(1-R)^{2}} & \text { for } & 0 \leq R<1
\end{array}\right.
$$

Additionally, it is important to know that refined mesh in the control volume around the notch tip is not necessary to determine the value of the SED, because this parameter can be determined via the nodal displacements, without involving their derivatives. This means that the adopted FE meshes can be very coarse inside the control volume having radius $R_{0}$ when accurately evaluating the value of the local SED [27].

The assumption of the SED as a damage parameter allows to summarize a large number of fatigue data obtained for notched specimens in a reasonable scatter band [10, 23, 28]. Generally, it is important to understand the fatigue behaviour of notched components and to assess the fatigue strength without performing a large number of experiments. Therefore, in order to predict the fatigue life of components having different geometrical features, the SED, $\Delta W$, versus the fatigue life, $N_{\mathrm{f}}$, relationship is calculated by considering the notched samples as sharp as possible. The averaged SED, $\Delta W$, can 
be obtained from the post-processing results by applying the value of nominal stress in the FE models. The relationship of the SED, $\Delta W$, and the fatigue life, $N_{\mathrm{f}}$, can be written as:

$$
\Delta W=A \cdot N_{\mathrm{f}}^{B}
$$

where $A$ and $B$ are material constants. Keeping constant the material and the nominal load ratio, the fatigue life can be assessed by using Eq. (6) for any geometrical configuration of the notch.

\section{THE SED TO ESTIMATE LIFETIME OF NOTCHED COMPONENTS UNDER VA FATIGUE LOADING}

$\mathrm{I}$

$\mathrm{n}$ the presence of VA load histories, the value of the SED will change as the amplitudes of the applied load spectrum vary. This clearly implies that, to correctly extend the use of the SED to those situations involving VA loadings, the procedure of the SED has to be redefined coherently according to the specific features of the assessed load spectrum.

When the notched sample is subjected to the VA fatigue loading, the VA load spectrum can be counted by the Rain-Flow method [20]. Such a spectrum is formed by $j$ different stress levels that are characterized by a nominal stress amplitude equal to $\sigma_{\mathrm{a}-\mathrm{i}}$ as shown in Fig. 4 . The corresponding number of cycles is equal to $\mathrm{n}_{i}(i=1,2, \ldots, j)$, where:

$$
n_{\mathrm{tot}}=\sum_{i=1}^{j} n_{i}
$$
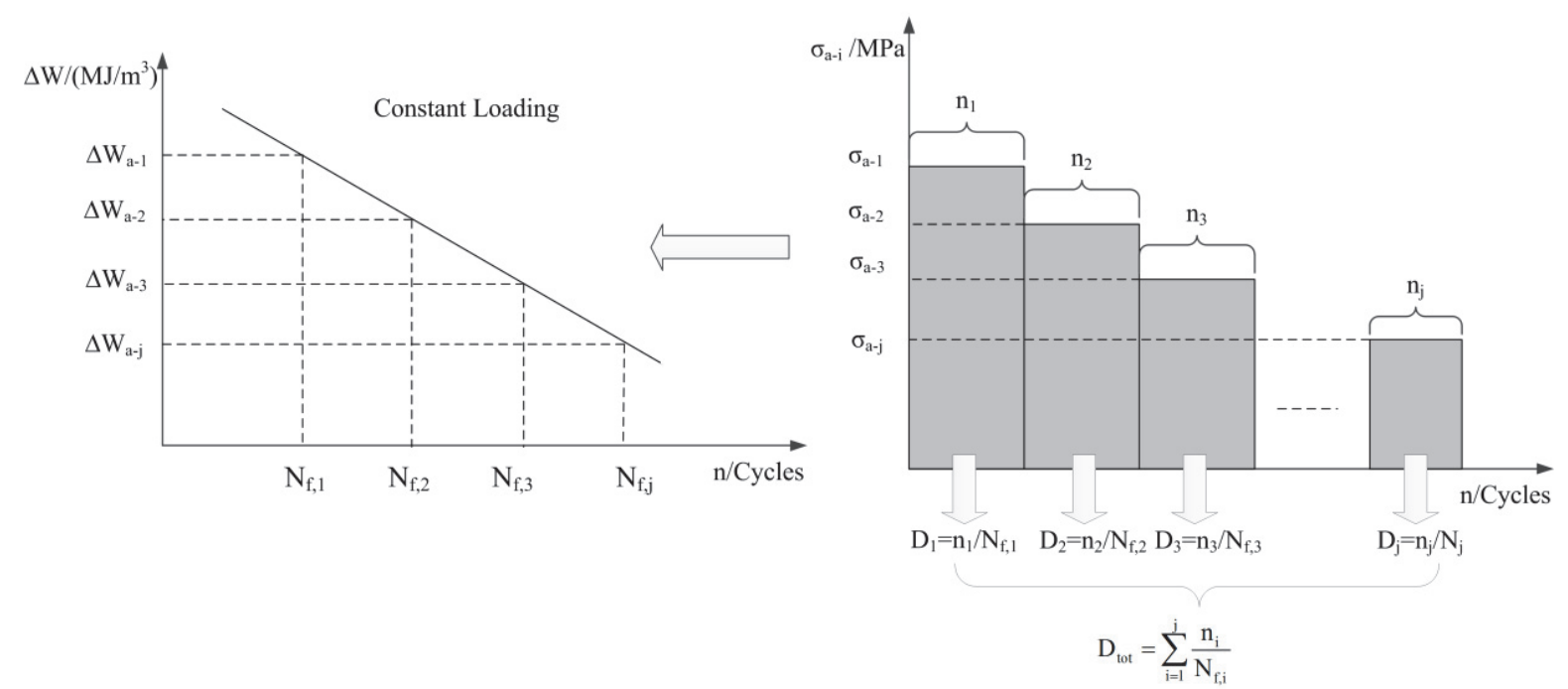

Figure 4: Estimation of the fatigue lifetime under VA loading by SED.

The different stress levels forming the above load spectrum can be treated as an independent CA loading, which can be applied in the FE model to obtain the value of the SED. Then, the corresponding number of cycles to failure $N_{\mathrm{f}, i}$ can directly be determined according to the relation of $\operatorname{SED} \Delta W$ versus the fatigue life $N_{\mathrm{f}}$ obtained by Eq. (6). As formalized by Palmgren and Miner [1,2], the fatigue damage content associated with any stress level can be calculated as follows:

$$
D_{i}=\frac{n_{i}}{N_{\mathrm{f}, i}}
$$

The resulting total damage is equal to: 


$$
D_{\text {tot }}=\sum_{\mathrm{i}=1}^{\mathrm{i}} \frac{n_{i}}{N_{\mathrm{f}, i}}
$$

Finally, the number of cycles to failure, $N_{\mathrm{f}, \mathrm{e}}$, can be estimated from the predicted number of blocks to failure, $N_{\mathrm{b}, \mathrm{e}}$, i.e.:

$$
N_{\mathrm{b}, \mathrm{e}}=\frac{D_{\mathrm{cr}}}{D_{\mathrm{tot}}} \Rightarrow N_{\mathrm{f}, \mathrm{e}}=n_{\mathrm{tot}} \cdot N_{\mathrm{b}, \mathrm{e}}
$$

where $D_{\mathrm{cr}}$ is the critical value of the damage sum.

\section{VALIDATION BY EXPERIMENTAL DATA}

7 he data used to validate the proposed extension of the SED approach are those generated by Susmel and Taylor by testing the notched samples of C40 under both the Concave upwards Spectrum (CUS) and Concave Downwards Spectrum (CDS) [3]. As to the strain energy density analysis, the cylindrical samples were modelled by using axisymmetric bi-dimensional model with coarse meshes inside the volume.

By applying the net nominal stress $\sigma_{\mathrm{An}}$ at $10^{6}$ cycles to the FE model, the numerical values of $\Delta W^{\text {notch }}$ as a function of the critical radius $R_{0}$ were obtained as shown in Fig. 5 . It is observed that the value of the critical radius $R_{0}$ is about $0.11 \mathrm{~mm}$ when the value of the SED for the V-notched specimen equals to that of plain specimen, $\Delta W_{0}^{\text {plain }}$.

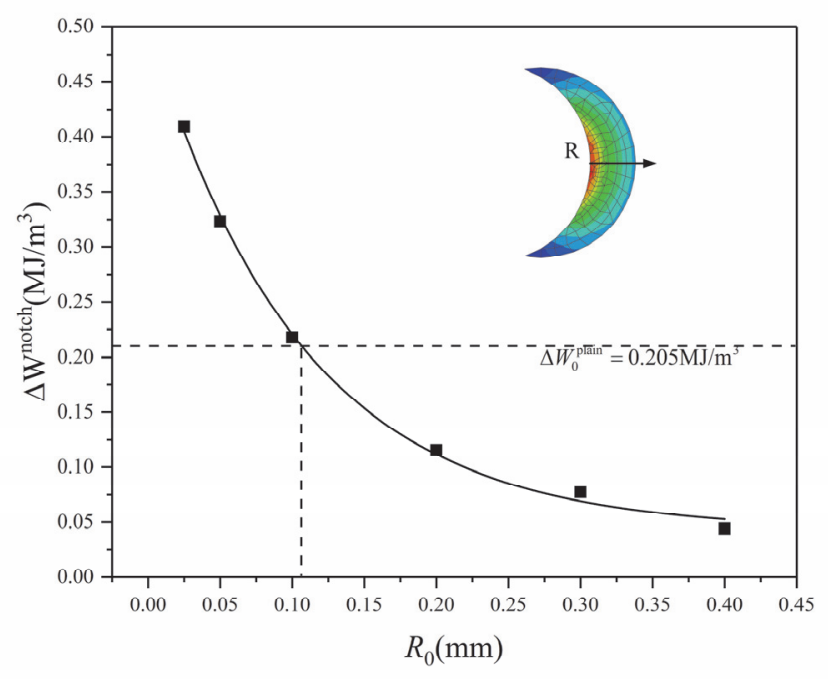

Figure 5: Numerical values of $\Delta W^{\text {notch }}$ as a function of the critical radius $R_{0}$.

The value of the SED averaged over the control volume was also calculated numerically by using the FE method. As shown in Fig. 4, a set of stress levels are acquired after calculating the load spectrum by Rain-Flow method. Then, the values of the SED of notched specimens with the sharpest radius are evaluated by applied above stress levels in the FE model. According to Eq. (6), the relationship of the $\operatorname{SED} \Delta \bar{W}$ versus fatigue life $N_{\mathrm{f}}$ under CA fatigue loading is

$$
W=242.4 \times N_{\mathrm{f}}^{-0.5266}
$$

where the constants in Eq. (10) are determined by a best fit to a series of calculated data.

As to the critical value of the damage sum, $D_{\text {cr }}$, initially the hypothesis was formed that it could be taken equal to its average value experimentally determined from the plain samples, $D_{\mathrm{cr}, \exp }=0.63$. The obtained results with different notch 
root radius are summarized in the experimental, $N_{\mathrm{f}}$, vs. estimated, $N_{\mathrm{f}, \mathrm{e}}$, fatigue lifetime diagrams reported in Fig. 6a. The predictions with small notch radius made using the SED are seen to fall always within the parent material scatter band. For the medium notch radius, the predicted results mostly fall within the patent material scatter band. The predictions with large notch radius nearly fall outside of the parent material scatter band, and on the non-conservative side. In this case, the accuracy of the predicted results is higher as the notch becomes sharper. As shown in Fig. 6a, the novel formalization of the SED is seen to be capable of accurately assessing the fatigue lifetime of notched specimens under VA loading by adopting simple Palmgren-Miner rule. Using this approach can reduce the time and costs associated with the design process.
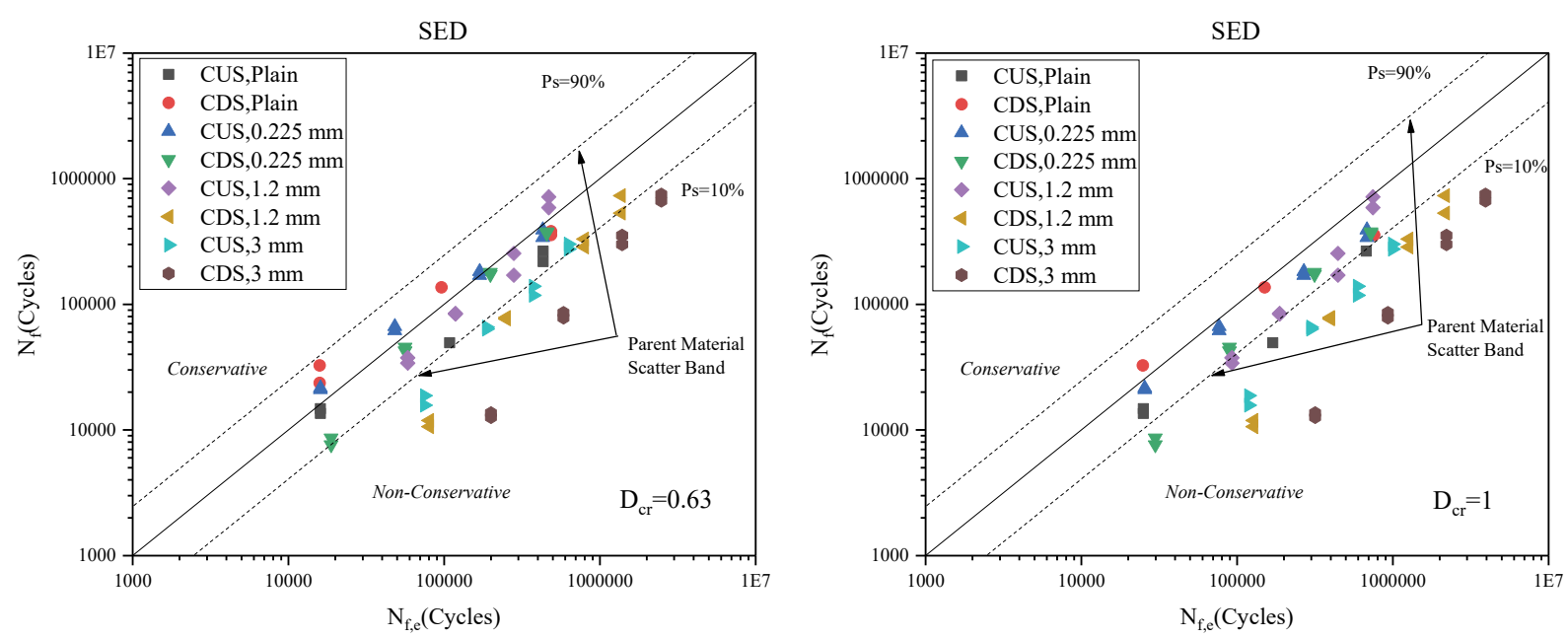

Figure 6: The accuracy of SED in estimating the fatigue lifetime of the notched samples under two critical value of the damage sum,

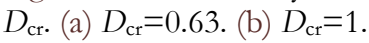

The same analysis as above was performed also by taking, as suggested by the classical theory by Palmgren and Miner, a critical value for the damage sum equal to unity. The diagram reported in Fig. $6 \mathrm{~b}$ shows that for the damage sum equal to unity, the level of non-conservatism of increases, resulting in poor accurate estimates. The predictions with small notch radius have the highest accurate estimates, but on the non-conservative side. Other predictions with medium and large notch radius mostly fall outside of the parent material scatter band and on the non-conservative side. It is also interesting to note that the estimated results under CUS are higher accuracy than the results under CDS, which shows that the loading shape has obvious effect on the fatigue behaviour. In the CDS, the cycles of low stress levels take up the majority of the sequence length, which would make the prediction a little inaccurate.

According to the experimental evidence (Tab. 5), the critical value $D_{\mathrm{cr}}$ of plain specimens varied as the shape of the load spectrum change. $D_{\text {cr }}$ under the CUS spectrum is lower than the value of critical damage sum under the CDS spectrum. Such a high variability shows that the critical value of critical damage sum strongly affects the accuracy of predicted fatigue lifetime. So far, the only way to correctly obtained $D_{\text {cr }}$ is by running enough experiments. Though many damage sum theories have also been developed [29-35], unfortunately, none of them enjoys universal acceptance due to the complexity of the problem, such as nonlinear damage, load sequence and small amplitude stress below fatigue limit. Consequently, the Palmgren-Miner rule is still dominantly used in design, in spite of its major shortcomings. More efforts on the cumulative damage are needed in order to provide design engineers with a general and reliable fatigue damage analysis and life prediction approach.

\section{CONCLUSIONS}

I $\mathrm{n}$ the present work, the fatigue criterion based on the strain energy density (SED) has been applied to re-analyse some experimental results reported in the recent literature. The specimens being considered were characterized by three notch tip radius and tested under uniaxial loading. The relationship of the averaged SED and the number of cycles to failure was calculated by considering the sharper specimens. According to above the relationship and the RainFlow method, the novel formalization of the SED proposed was seen to be accurate in estimating fatigue lifetime of 
notched components damaged by VA uniaxial fatigue loading. It has also been observed that the critical value of damage sum has obvious effects on the accuracy of estimated fatigue life.

\section{ACKNOWLEDGEMENTS}

7 he authors would like to acknowledge the support by the Innovation Program (237099000000170004) and the State Key Laboratory Program (614221305020617) and also by the China Scholarship Council (Grant No. 201705290009).

\section{REFERENCES}

[1] Palmgren A. (1924). Durability of ball bearings, ZVDI, 68(14), pp. 339-341.

[2] Miner M. (1945). Cumulative Damage in Fatigue, J. Appl. Mech., 12, pp. A159-A164.

[3] Susmel L., Taylor D. (2011). The Theory of Critical Distances to estimate lifetime of notched components subjected to variable amplitude uniaxial fatigue loading, Int. J. Fatigue., 33(7), pp. 900-911.

[4] Susmel L, Taylor D. (2012). A critical distance/plane method to estimate finite life of notched components under variable amplitude uniaxial/multiaxial fatigue loading, Int. J. Fatigue., 38, pp. 7-24.

[5] Neuber H. (1958). Theory of notch stresses: principles for exact calculation of strength with reference to structural form and material, 2nd ed.. Berlin: Springer Verlag.

[6] Molski K., Glinka G. (1981). A method of elastic-plastic stress and strain calculation at a notch root, Mater. Sci. Eng., 50(1), pp. 93-100.

[7] Ellyin F., Kujawski D. (1989). Generalization of notch analysis and its extension to cyclic loading, Eng. Fract. Mech., 32(5), pp. 819-826.

[8] Lazzarin P., Zambardi R. (2001). A finite-volume-energy based approach to predict the static and fatigue behavior of components with sharp V-shaped notches, Int. J. Fracture., 112(3), pp. 275-298.

[9] Taylor D. (1999). Geometrical effects in fatigue: a unifying theoretical model, Int. J. Fatigue., 21(5), pp. 413-420.

[10] Lazzarin P., Berto F. (2005). Some Expressions for the Strain Energy in a Finite Volume Surrounding the Root of Blunt V-notches, Int. J. Fracture., 135(1-4), pp. 161-185.

[11] Lazzarin P., Berto F. (2008). Control volumes and strain energy density under small and large scale yielding due to tension and torsion loading, Fatigue. Fract. Eng. M., 31(1), pp. 95-107.

[12] Berto F., Lazzarin P. (2013). Fatigue strength of Al7075 notched plates based on the local SED averaged over a control volume, Sci. China. Phys. Mech., 57(1), pp. 30-38.

[13] Berto F., Gallo P., Lazzarin P. (2014). High temperature fatigue tests of un-notched and notched specimens made of 40CrMoV13.9 steel, Mater. Design., 63, pp. 609-619.

[14] Negru R., Șerban D., Marșavina L., Magda A. (2016). Lifetime prediction in medium-cycle fatigue regime of notched specimens, Theor. Appl. Fract. Mec, 84, pp. 140-148.

[15] Berto F., Lazzarin P., Yates J.R. (2011). Multiaxial fatigue of V-notched steel specimens: a non-conventional application of the local energy method, Fatigue. Fract. Eng. M., 34(11), pp. 921-943.

[16] Berto F., Lazzarin P. (2011). Fatigue strength of structural components under multi-axial loading in terms of local energy density averaged on a control volume, Int. J. Fatigue., 33(8), pp. 1055-1065.

[17] Berto F., Campagnolo A., Lazzarin P. (2015). Fatigue strength of severely notched specimens made of Ti-6Al-4V under multiaxial loading, Fatigue. Fract. Eng. M., 38(5), pp. 503-517.

[18] Berto F., Gagani A., Aversa R., Petrescu R.V.V., Apicella A., Petrescu F.I.T. (2016). Multiaxial fatigue strength to notched specimens made of 40CrMoV13. 9, Am. J. Eng. Appl. Sci., 9(4), pp. 1269-1291.

[19] Berto F., Lazzarin P., Yates J. (2011). Multiaxial fatigue of V - notched steel specimens: a non - conventional application of the local energy method, Fatigue. Fract. Eng. M., 34(11), pp. 921-943.

[20] Matsuishi M., Endo T. (1968). Fatigue of metals subjected to varying stress, Japan Society of Mechanical Engineers, Fukuoka, Japan, 68(2), pp. 37-40.

[21] Gurney T.R. (2006). Cumulative damage of welded joints, Woodhead Publishing.

[22] Lazzarin P., Campagnolo A., Berto F. (2014). A comparison among some recent energy- and stress-based criteria for the fracture assessment of sharp V-notched components under Mode I loading, Theor. Appl. Fract. Mec, 71, pp. 21 30. 
[23] Berto F., Lazzarin P. (2014). Recent developments in brittle and quasi-brittle failure assessment of engineering materials by means of local approaches, Materials Science and Engineering: R: Reports, 75, pp. 1-48.

[24] Razavi S., Ferro P., Berto F., Torgersen J. (2018). Fatigue strength of blunt V-notched specimens produced by selective laser melting of Ti-6Al-4V, Theor. Appl. Fract. Mec, 97, pp. 376-384.

[25] Lazzarin P., Livieri P., Berto F., Zappalorto M. (2008). Local strain energy density and fatigue strength of welded joints under uniaxial and multiaxial loading, Eng. Fract. Mech., 75(7), pp. 1875-1889.

[26] Lazzarin P., Sonsino C., Zambardi R. (2004). A notch stress intensity approach to assess the multiaxial fatigue strength of welded tube - to - flange joints subjected to combined loadings, Fatigue. Fract. Eng. M., 27(2), pp. 127-140.

[27] Lazzarin P., Berto F., Zappalorto M. (2010). Rapid calculations of notch stress intensity factors based on averaged strain energy density from coarse meshes: Theoretical bases and applications, Int. J. Fatigue., 32(10), pp. 1559-1567.

[28] Berto F. (2016). Fatigue and fracture assessment of notched components by means of the Strain Energy Density, Eng. Fract. Mech., 167, pp. 176-187.

[29] Manson S., Halford G.R. (1981). Practical implementation of the double linear damage rule and damage curve approach for treating cumulative fatigue damage, Int. J. Fracture., 17(2), pp. 169-192.

[30] Halford G.R. (1997). Cumulative fatigue damage modeling-crack nucleation and early growth, Int. J. Fatigue., 19(93), pp. 253-260.

[31] Fatemi A., Yang L. (1998). Cumulative fatigue damage and life prediction theories: a survey of the state of the art for homogeneous materials, Int. J. Fatigue., 20(1), pp. 9-34.

[32] Socie D. (1987). Multiaxial fatigue damage models, J. Eng. Mater. Technol., 109(4), pp. 293-298.

[33] Lagoda T. (2001). Energy models for fatigue life estimation under uniaxial random loading. Part I: The model elaboration, Int. J. Fatigue., 23(6), pp. 467-480.

[34] Bui-Quoc T. (1982). Cumulative damage with interaction effect due to fatigue under torsion loading, Exp. Mech., 22(5), pp. 180-187.

[35] Aïd A., Amrouche A., Bouiadjra B.B., Benguediab M., Mesmacque G. (2011). Fatigue life prediction under variable loading based on a new damage model, Mater. Design., 32(1), pp. 183-191. 